\title{
LES FEDERATIONS RADJA NA IX ET RADJA NA X DE LA COTE EST DE SUMATRA
}

\author{
G. L. Tichelman
}

\section{LE CAD'RE GEOGRAPHIQUE}

1. Situation. Dans le sud d'Asahan se trouvent les anciennes fédérations Radja na Sembilan et Radja na Sepoulouh ayant pour place principale Rantau Prapat; leur superficie est de $385 \mathrm{~km}^{2}$ environ, et jusqu'ici les descriptions de cette contrée peu visitée sont rares.

Joustra ${ }^{1}$ nomme les indigènes «Bataks en dehors du pays Batak» et relève qu'on y retrouve les relations sociales des Bataks. La population étant devenue musulmane, son sentiment de parenté avec les Bataks proprement dits, a diminué.

2. Terrain. Les' deux fédérations ${ }^{2}$ à la base du massif du Bukit Barisan sont en grande partie montagneuses et fort boisées. En venant de Bilah par Kouala Aëk Pandan on rencontre de grandes plaines, qui avec quelques collines, s'étendent vers le sud ouest ${ }^{3}$. Le terrain monte; à Motong Oulou on se trouve en pleine montagne et en croisant la crête du Tor Kompas, on passe dans la Radja na Sepoulouh, où le premier village Masiki Ilir est à $140 \mathrm{~m}$ au dessus du niveau de la mer. Par un terrain très accidenté on arrive à Sopolongat Oulou, la plus ancienne station selon la tradition locale. Vers l'ouest, Batou Djongdjong se trouve dans les montagnes, Kouala Masihi dans la plaine basse. Le passage du Tor Langgeh entre Hatapang Oulou et Djapadang est difficile. Les principaux sommets sont le Tor Kompas, le Tor Gonting Sorik, le Tor Langgeh et le Tor Batou Sinorpi qui monte à plus de 1000 mètres.

3. Rivières. La fédération Radja na Sembilan se trouve en amont de la Marbau qui, avec les Aëk Ketiak et Pamengke, est tributaire de gauche de la rivière Bilah. Dans le Dolok, le confluent de l'Aëk Paningaän et de l'Aëk Persagouan s'appelle Aëk Kota Batou, jusqu'à Poulau Hopour et dès ce point Aëk Marbau. Elle reçoit de nombreuses rivières, dont les principales à droite sont l'Aëk Hadjoran, l'Aëk Hatapang et l'Aëk Pandan, ayant à leurs embouchures respectives Adaren, Djampahan et Kouala Aëk. Les autres principaux villages le long de la Marbau sont Marbau et Si Pare Pare ${ }^{*}$. A deux heures de marche en aval de Si Pare Pare, la Marbau se jette dans la Bilah, qui à son tour, en aval du village Labouan Bilik, débouche dans la Panei.

La Bilah et la Marbau sont navigables pour vapeurs à petit tirant d'eau jusqu'à Pare Pare. Des prahous jaugeant 45 pikols montent jusqu'à Kouala Marbau, et en amont de ce poste dépendant du niveau d'eau, des pirogues découpées dans un tronc d'arbre, contenant de 2 à 5 pikols (180 à 300 kilos) peuvent pousser jusqu'à Kouala Pandan.

La Radja na Sepoulouh fait partie de la région en amont de la Bilah par l'Aëk Ketiak et l'Aëk Pemengke (ou Balamengke) qui reçoivent de nombreux petits cours d'eau montagneux, sans importance pour le transport.

La présence des rivières rend la contrée fertile et permet leur exploitation dans les vallées. Jusqu'au début de la guerre, la population y vivait relativement prospère.

4. Climat. Aux journées chaudes succèdent généralement des nuits fraiches, même froide. La courte saison des pluies compte avril et mai, la grande saison des pluies octobre, novembre et décembre. Juin et juillet sont la période la plus sèche.

\section{PEUPLEMENT ET CIVILISATION}

5. Routes. Un tracé non pavé de 2 à 3 mètres de large, avec quelques petits ponts de bois, reliait les villages; en 1932 une route asphaltée fut ouverte. Dans le terrain difficile, certains postes ne sont reliés que par des sentiers. Tout transport terrestre se fait par porteurs, les charges se portent sur la tête ou le dos dans des paniers (v). De Masihit Ilir à Masdjid ou à Si Poldung on compte 3 jours de marche, à Si Bouhouan 4 jours, à Marbau 7 hẹures.

1 Joustra, "Batakspiegel ", 2ème éd. 1926, pag. 344, 345.

2 Le Radja na Sembilan comprend: Oudjoung Godang, Pasang Lela, Poulau Godang, Poulau Hopour, Tardas, Montong, Djapadang, Hatapang Oulou, Bourangir; - le Radja na Sepoulouh: Masihi Ilir, Masihi Oulou, Rimbaia, Kouala Masihi, Batou Tounggal, Houta Barou, Aëk Kopong, Padang Nabidang, Napompar, Batou Djondjong.

3 Oudjoung Godang, Pasang Lela, Poulau Godang, Poulau Hopour, Tardas.

* Hadjoran Oulou, Adaran, Hatogouan, Telok Godang, Poulau Hopour, Poulau Godang, Kouala Aëk Pandan, Ilir. 


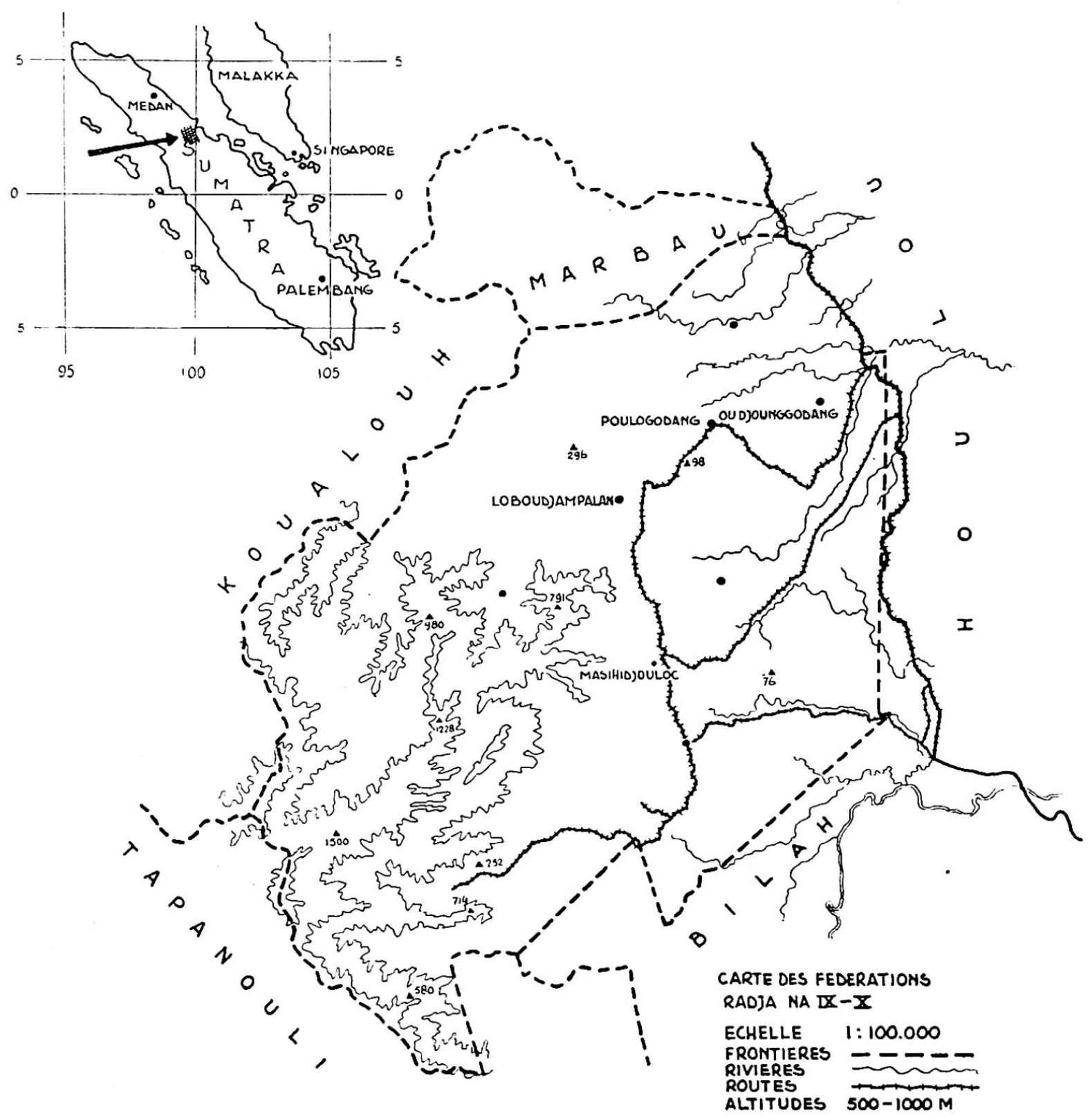

6. Villages. Autrefois il n'y avait que des habitations éparses sur les ladangs (v); sous l'influence des premiers fonctionnaires civils les agglomérations se sont formées au début du siècle, en général une rangée de maisons le long des rivières.

Construites en matériel léger comme chez les malais, les huttes des paysans sont placées sur de minces poteaux à 5 ou 6 pieds au dessus du sol. Le plancher est en bois de niboung, avec des trous par lesquels on dépose les selles et ordures, les parois sont en écorce d'arbre et ont des trous pour permettre de voir, la toiture est en fibre idjouk (v), souvent en alang (v). Un mince tronc d'arbre muni d'entailles conduit comme une échelle à la porte d'entrée.

Les maisons des chefs, Ketoua ou Penghoulou (v), sont bâties d'ordinaire dans le style Toba, à 5 ou 6 pieds au dessus du sol, sur de lourds poteaux en bois reposant sur des assises en pierre. Le plancher et les parois sont en bois. Une porte avec échelle ou escalier se trouve dans la paroi frontale, des fenêtres munies de volets dans les parois latérales, qui s'élèvent légèrement penchées vers le dehors. La toiture haute et raide est ordinairement couverte d'idjouk (v), plus rarement de sirap (v). La maison ne consiste qu'en un seul appartement mal éclairé. Dans la partie arrière se trouve une caisse en bois, remplie d'argile: le foyer. Pour chaque ménage habitant la maison, il y a un foyer séparé, au dessus duquel sont mises des lattes pour le 
séchage. D'autres étagères se trouvent par ci par là pour divers ustensiles. La paroi frontale regardant vers la rue, est la moins longue.

$\mathrm{V}$ is à vis de la maison du chef se trouve la sopo ( $\mathrm{v}$ ), une maison commune, salle de réunion, dortoir pour jeunes hommes et logement pour étrangers. Parfois c'est le plus grand bâtiment du village, habituellement sans parois, avec un foyer au milieu du plancher où un feu brûle pendant la nuit.

Les maisons de prière, mendersah $(\mathrm{v})$, sont bâties sur poteaux. Le plancher et les parois sont en bois, le toit est couvert d'idjouk (v).

7. Vêtements. Les hommes portent le pantalon, la chemise courte, le sarong ( $\mathrm{v})$ et soit le couvre-chef empesé soit une calotte $(\mathrm{v})$. Les cheveux sont coupés courts. Pour les occasions solennelles, les chefs portent veston noir et souliers. Les femmes portent à Tandjong Slamat le couvre-chef des Karo Batak, mais en couleurs vives. Elles portent le sarong long et une veste; les enfants, excepté aux jours de fête, courent nus les premières années, une amulette au cou pour les protéger contre les influences néfastes.

La parure pour hommes et femmes consiste en anneaux. Les femmes portent aussi des pendeloques aux oreilles, des colliers en perles, des bracelets d'argent ou de cuivre.

8. Nourriture. La nourriture principale est le riz, cuit à l'eau, sans sel. En temps de pénurie on y mêle du maïs. A part cela, on mange la cassave. On ajoute des légumes ou feuilles bouillies, des concombres avec du piment.

Le poisson et la viande de cerf se mangent rarement, le poulet est servi aux occasions spéciales. La boisson ordinaire est l'eau; le suc du palmier arène se mange avec le riz cuit ou se boit comme sirop. On récolte le vin de palmier qui se boit non fermenté (doux); il n'y a pas d'abus. Le café est rare.

L'entière population mâle fume: les jeunes garçons pouvant à peine marcher fument déjà des cigarettes de tabac roulé dans les bractées de maïs séchées. On n'emploie ni la pipe, ni l'opium; l'usage du sirih est très répandu.

9. Fêtes. L'orchestre comprend des tambours, une flûte et deux cymbales. Hommes et femmes de tout rang dansent en l'honneur de visiteurs d'importance. Les pieds glissent sur le sol, les bras sont tendus ou courbés à angle droit, les mains montrent la paume et alternativement la détournent. En général les femmes sont plus lentes, moins accoutumées.

Il y a également une danse des jeunes qui se fait avec l'accompagnement des tambours, agitant le slendang (v) avec lenteur, tenant les yeux modestement baissés. Cette danse doit être réciproquée par le chef ou son représentant, ce qui se fait aux cris de «salut». Comme témoignage d'appréciation on donne à la danseuse ou au danseur un sarong.

La danse des sabres se fait aux occasions spéciales: il s'agit de faire adroitement les gestes d'attaque et de défense de façon à ne pas se blesser, tout comme on le fait dans les autres îles de l'archipel. D'habitude les danseurs se donnent la main après la partie.

10. Politesse. Le bétel joue un rôle considérable. Les deux sexes l'emploient. On l'offre à chaque visiteur. Si celui-ci est un personnage important, par exemple le chef du district ou le sultan, on offre la boîte à bétel enveloppée dans un morceau de soie jaune, au toungkou besar (l'héritier présomptif du sultan) dans de la soie jaune et noire, aux datouks, dans de la soie noire. En acceptant la boîte, le visiteur doit déplier la soie, après quoi il suffira de prendre un menu morceau de pinang. Parfois la présentation du bétel est accompagnée de quelques œufs ou d'une corbeille de riz, ce qu'il faut accepter sans essayer de rendre un cadeau: ceci irait à l'encontre de l'adat ( $v$ ) et serait considéré comme une offense. 


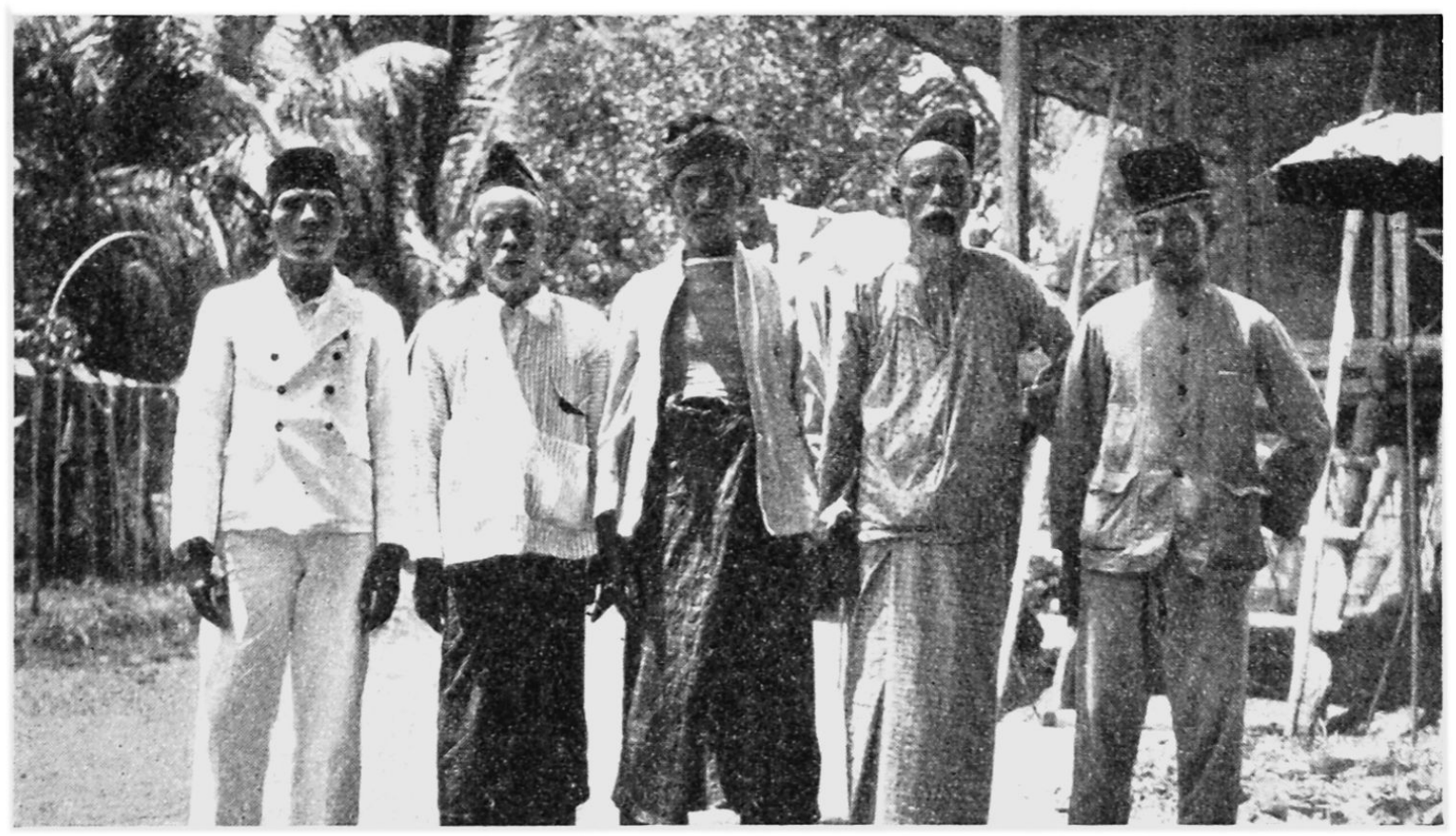

Notables du village Rimbaia (Radja na IX et na X), Photo Institut Colonial, Amsterdam.

11. Race. Selon la tradition, les Toba-Bataks sont partis de leur pays il y a sept «sunduts » ou générations, donc il y a à peu près deux siècles; le groupe actuel Radja na Sepoulouh a pris la direction sud-est, le groupe Radja na Sembilan est resté dans la contrée de la Marbau. On dit que le plus ancien établissement du Radja na Sepoulouh est Sopolangat Oulou; les gens disent être venus de Muara Toba, marga Ritonga. Vers 1890, Hadji Abdœrachman de Padang Sidempuan s'établit à Pasang Lela et prêcha l'Islam à la population païenne. En 1934, il y vivait encore, fort âgé et très respecté.

Les gens de Napompar sont venus, disent-ils, de Pakpak, Daïri, avant la naissance de la grand'mère du chef (qui était nonagénaire en 1932).

Chaque Batak connaît sa marga ${ }^{5}$ ou généalogie et chaque radja possède une liste qu'il garde soigneusement dans un bambou. Les margas vivent cependant entremêlés.

Les types populaires à Napompar et à Nabidang ont en général des traits assez grossiers, le crâne long, le front peu tombant, le nez long, large et applati, la lèvre supérieure longue, les mâchoires saillantes, le menton court, la bouche large, les lèvres grosses, les pomettes saillantes, les oreilles détachées. Il n'y a que peu de barbe. La couleur de la peau est brun jaunâtre; les yeux bruns foncé, grands et un peu élargis, montent légèrement vers les tempes. Les sourcils sont gros et bien marqués, les cheveux noirs et lisses sont portés en torsade par les femmes. La taille est courte (environ $1 \mathrm{~m} \mathrm{65}$ ), assez trapue, la musculature est assez faible, les jointures grosses, les mains et les pieds larges, les doigts courts.

En 1938 le nombre d'habitants était de 5783, avec une légère prépondérance masculine.

12. Religion. Sous l'influence de la Mission de Tapanouli, quelques ménages à Sopolangat Oulou se sont convertis au christianisme; le reste de la population professe l'Islam. Sans être fanatique on pratique les prières journalières.

${ }^{5}$ Les margas sont Dadi-Maunté, Pohan, Pahoutar, Sagala-Ritonga, Hasibouan, Rambé, Harahap, Nasoution, Dairi, Dongoran, Sim Sim, Simatoupang, Bakkara, Samosir, Simondjountak, Simbolon, Dasopang, Pospos, Batou Bara et Tandjoung. 
Il y a presque toujours une maison de prière (mendersah ou souran) et un prédicateur (malim ou bebei), mais pas de missigit ( $v$ ). Le Batak converti à l'Islam se considère comme devenu malais et souvent se croit insulté par l'appellation d' «orang Batak». Cependant aux grandes occasions, mariages, réconvalescences, longs voyages, etc., on pratique la cérémonie oupar (prière de bénédiction, repas d'offrande). Chaque convive reçoit une tourelle de riz garnie de viande et d'œufs durs, parfois colorés et décorés d'un arbre de vie ${ }^{6}$ couronné par l'oiseau du paradis, artistement façonné en papier coloré. A ces occasions, on offre un poulet, une chèvre ou un buffle. Si l'oupa oupa (v) se fait en faveur d'un enfant, on lui enduit le front du jaune d'œuf, symbole du principe vital.

A la naissance d'un enfant on le baigne presque immédiatement dans la rivière et donne ensuite un repas de fête. Sur demande, le prédicateur donne à l'enfant un nom qu'il garde, à moins que des maladies réitérées ne rendent le changement souhaitable. Vers la puberté, les dents doivent êtres limées chez les filles comme chez les garçons. Peu après, la circoncision se fait chez ces derniers par le malim et chez les jeunes filles par la malim daborou, c. à. d. la sage-femme qui, entre autre, lave les défuntes. Le garçon devenu jeune homme ne dormira plus chez ses parents mais dans la sopo (v). Les amants se servent de symboles: une touffe de feuilles d'oubi veut dire « je suis pauvre, mais je t'aime»; le fruit de l'ananas, déposé à un lieu de rencontre et une pirogue découpée dans un morceau de bois indiquent des chansons populaires (pantoungs) ${ }^{7}$.

Le jeune homme qui veut se marier part à la recherche d'une femme, s'il a une jeune fille en vue, il envoie un émissaire pour délibérer avec les parents. Si l'on se trouve d'accord, la dot (boli) est fixée, le futur gendre apporte des cadeaux à ses beaux-parents, et une date favorable est choisie. Le fiancé paie les frais et la dot, remise en son entièreté, lui donne le droit d'emmener sa femme à son village. Le mariage est patrilocal. Tant que la dot n'est pas complètement payée, le jeune homme reste chez ses beaux-parents. Les cadeaux à la fiancée se soustraient du montant de la dot. Dans le premier quart du siècle, la dot variait de 20 à 65 florins, pour les filles des chefs de 200 à 300 florins.

Par ostentation on annonce souvent un montant plus haut que celui qui se paie. Tant que la vie conjugale est harmonieuse, on ne parle plus du restant. Quand il y a divorce ou querelle avec les parents, la dot est réclamée.

Si les parents de la jeune fille s'opposent au mariage, celui-ci peut se faire après enlèvement (lompat pagar). Ces mariages sont conclus dans la maison du malim. La boli réclamée est payée aux parents en plus de la moitié du montant pour apaiser la colère. La moitié de cette toulak sendjata (v) revient au chef. Le mari aussi bien que la femme peuvent demander le divorce; celui qui l'exige est considéré comme ayant tort (salah): le mari divorcé cède la boli payée, et si la femme demande le divorce, la dot est repayée par la famille. La mort de la femme ne décharge pas le mari de l'obligation de payer la dot. Comme les mariages sont coûteux, la polygamie est restreinte aux chefs et aux riches; il n'y a pratiquement pas d'adultère.

${ }^{6}$ On peut y comparer l'Hindouisme, l'arbre céleste comme figure centrale du wajang, et la barque des esprits de Célèbes.

7 les deux pantoungs notés sont:

« Bounga nanas, bounga tjempaga,

* Dimana dapat sama kembangnja,

* Adinda omas, saja tombaga,

* Dimana rapat, sama timbangnja *.

La fleur de l'ananas et du tjempaka

Sont les mêmes fleurs partout où on les trouve,

Ma sœur est d'or, je suis de cuivre,

Mais là où elles se rencontrent, elles pèsent la même chose.

* Kapal Bongali terlalou sarat,

* Orang Arab poulang ke Djouddah,

" Bosar pahalka menjampaikan harap,

* Harapnja saja boukannja moudah *.

Le navire du Bengal est petit,

L'Arabe retourne à Djeddah,

Que les grands dieux aident mon espoir,

Car ce que j'espère, n'est pas chose facile. 
Les mariages dans la marga sont défendus. On n'accepte pas la coutume de certains districts Bataks où on peut donner une offrande en compensation de l'inceste par mariage dans la marga. Au décès du mari, son frère cadet mariera la veuve, sinon les enfants nés du mariage reviendront à la famille du mari dès qu'ils auront atteint 7 ans.

Les enfants de deux frères ne peuvent se marier, les mariages des enfants de frère et sœur sont admis, si le frère a une fille et la sœur un garçon ( «cross-cousin mariage»). Dans le cas contraire, le mariage est défendu. Mais on dit que cette coutume va bientôt disparaître. Le droit individuel mahométan supplante le droit familial.

Le bétel joue un rôle dans les cérémonies du mariage: à la demande en mariage, on offre la boîte de bétel; si, le jour de mariage, la femme paraît n'être pas vierge, les parents du mari portent la boîte à bétel fermée aux parents de la femme, qui, en ouvrant la boîte, constatent avec peine que les couvercles manquent aux petites boîtes en cuivre, ce qui peut occasionner de vives querelles.

Les morts sont enterrés de façon musulmane. La veuve suivait son fils ainé, plus récemment elle pouvait retourner chez sa famille, et en tous cas, elle a droit à ses effets personnels. La division de l'héritage se fait de concert. Le fils aîné hérite la maison. S'il y a deux fils, l'aîné reçoit les deux tiers, le cadet un tiers des possessions. Les filles ne reçoivent que leurs effets personnels. Il est très rare qu'il n'y ait pas de partage. Il n'y a pas de droit sur le sol, mais bien sur ce qui y est planté. Si la veuve se remarie, elle conserve le droit sur sa part de l'héritage, c'est un droit personnel.

13. Droit. Les chefs jugent les menus litiges, avec appel à la cour du radja avec les datouks et des fonctionnaires du kerapatan (v). Ces sessions se tiennent dans la sopo. L'ancienne cour d'appel supérieure, le kerapatan besar, formée par les radjas d'une des fédérations est tombée en désuétude par suite des jalousies des radjac. Après la pénétration de l'administration néerlandaise, les condamnés de la kerapatan prirent coutume d'adresser leur appel au fonctionnaire civil à Labouan Batou, ce qui amena l'organisation successive de tribunaux de districts siégeant à tour de róle. Ces tribunaux jugeaient les menus litiges allant jusqu'à 100 florins d'avant guerre, où le cas pénal entraînait une amende maximum de 25 florins, soit un mois de détention. La cour d'appel, kerapatan ketjil, et la cour d'appel supérieure, le kerapatan besar, se tenaient à Negri Lama. La révolution sociale de 1946 a aboli ces cours de justice, comme toutes les institutions dites féodales. On ne connaissait pas de jugement de Dieu; les crimes étaient rares, le juge avait en général à traiter les cas d'insubordination, le manque d'égards vis-à-vis des chefs, le payement de prêts, de bolis, etc. Comme punition on employait l'amende, dont une moitié revenait au juge, l'autre à la partie préjudiciée.

14. Langue. Dans les fédérations, on parle la bahasa oulou, un dialecte Batak parenté au Batak Angola et le Koualouh. Pour le contact avec les peuples voisins, on emploie le Batak Toba.

15. Armes. Il y a de vieilles armes à feu, fusils à pierre, pistolets, espingoles, etc. On emploie la poudre des feux d'artifice (mertjon) et achète le plomb pour les balles. En plus de cela, il y a la lance, le sabre, le kris et le couperet. Le peuple est pacifique, les villages ne sont pas fortifiés, les rares querelles aboutissaient à plus de tapage que de dommage. Les armes à feu sont surtout des marques de richesse, et employées contre les tigres, les sangliers et pour la chasse au cerf.

16. Hygiène. Les maladies cutanées, la dysenterie, la malaria et le struma sont fréquents. Les vieillards sont rares, en 1932 il y avait cependant à Napompar deux hommes et la grand'mère du chef, âgés de 90 ans. On raconte que longtemps avant la venue de l'Islam les vieillards étaient engraissés et après le décès, leur corps partagé entre les parents (endo-cannibalisme). Quand il survenait trop de maladies, de mauvaises récoltes, de catastrophes, on déménageait le village. Sur le nouveau terrain 


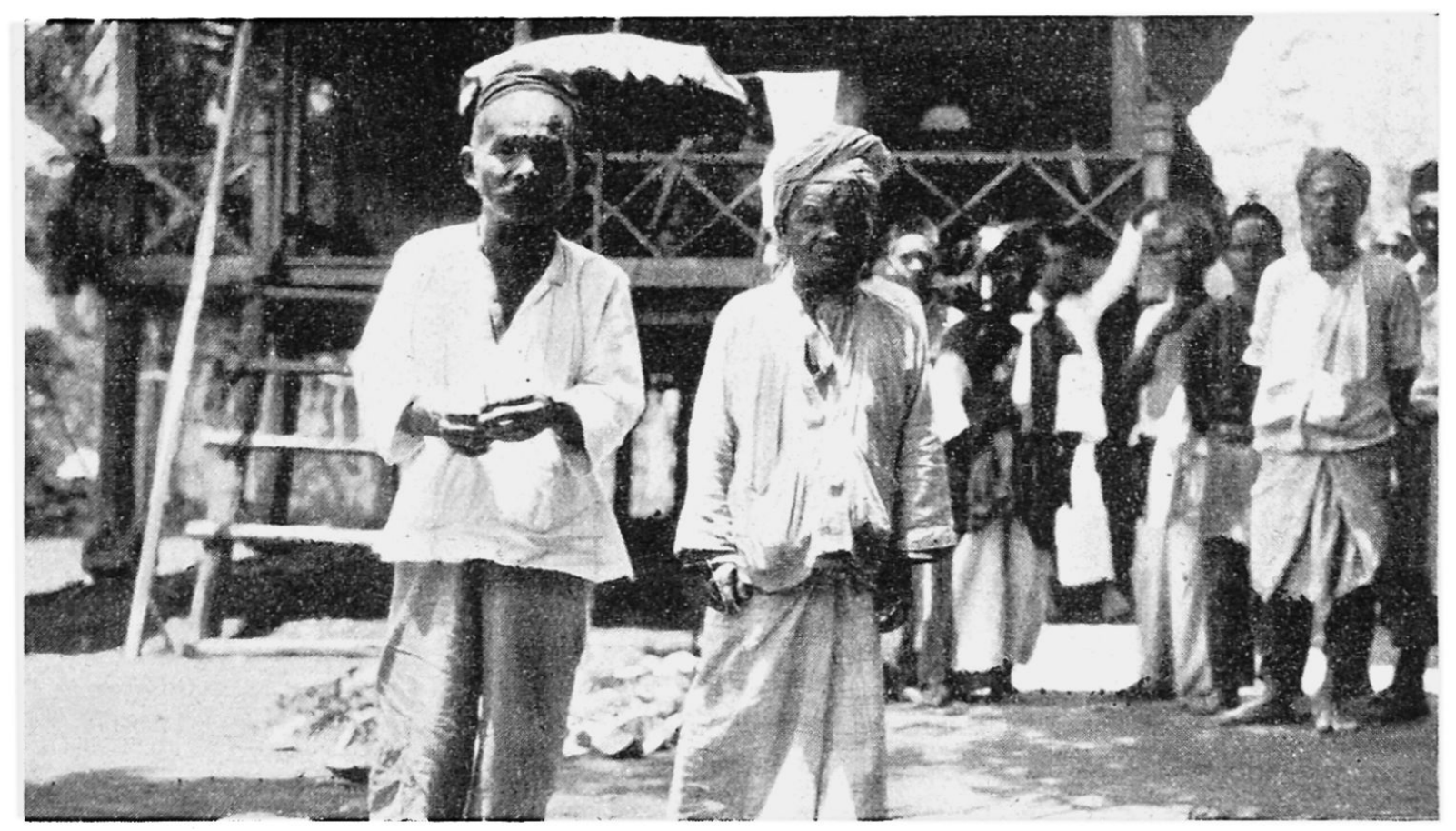

Nains d'un village Batak de l'arrière-pays d'Asahan. Photo Institut Colonial, Amsterdam.

on traçait un cercle et enfonçait un pieu dans la terre. La terre provenant du trou était semée sur le pourtour du cercle. Un coq, lié avec une patte au pieu, était sacrifié et on lui demandait de montrer avant de mourir l'endroit du nouveau village. La place où l'animal mourait indiquait cette direction.

17. Instruction. Les malims instruisaient les garçons dans la religion, d'abord par récitation du Koran, plus tard par sa lecture. A part cela, il y a eu quelques tentatives d'établir des écoles ${ }^{8}$.

18. Economie. Le plus important moyen d'existence est la culture sèche du riz. La culture des rizières fut stimulée par les fonctionnaires civils, mais pendant la guerre, la population s'est réfugiée dans les montagnes et la culture du ladang s'est accrue de nouveau. Pour le ladang on déblaie le terrain sans labourer: on sème quelques graines de riz dans de petits trous fait avec un plantoir. Entre le semage et la récolte, on ne fait que sarcler, arracher les mauvaises herbes et chasser les oiseaux et bêtes. Le dur labeur est fait par les hommes, femmes et enfants participent au reste. On plante en août et septembre et récolte en janvier et février en coupant le padi mur avec ses ongles, sans employer de couteau. La récolte reste entassée dans les huttes sur le ladang, sans crainte de vol. On la transporte par petites portions au village pour le battage qui se fait en piétinant. Avec de vieux essieux d'auto, les indigènes contruisirent des moulins à riz mis par l'eau courante.

Le maïs important aussi, souvent planté parmi le riz, mûrit en une centaine de jours. De même l'oubi et la cassave. La noix de cocotier est souvent récoltée par des singes dressés à cet effet. Presque chaque village a une petite plantation de tabac, pour le besoin propre. On élève quelques buffles, quelques chèvres, un cheval.

Il y a des plantations de café (Liberia) vendu à Rantau Prapat. Deci delà il y a des plantations indigènes de Hevea, qui deviennent de plus en plus importantes pour l'économie: en 1938 il y avait 731 plantations avec une production nominale de 75 tonnes par an; on livre le caoutchouc en slabs transportés par radeau à la dérive.

${ }^{8}$ Des écoles pour 40 à 50 garçons furent établies à Houta Barou et Poulau Hopour, des écoles de mission méthodiste à Padang Nabidang et Aëk Kota Barou et quelques écoles libres. 
La récolte du rotan a quelque importance de même que celle du getah percha.

Dans la forêt vierge, on trouve le bois pour construire les maisons, les bateaux, les poteaux. Dans la forêt jeune, on trouve les écorces de laque de la tachardia lacca en quantités de 2 à 3 kati. On l'emploie surtout pour le montage des hachoirs dans la manche. On ne connaît pas la culture de la laque comme à Sipirok et à India.

On chasse le cerf et les porcs qui abondent; les tigres sont rares, il n'y a pas d'éléphants. Les rivières ne sont pas riches en poissons, on y pêche l'ikan merah et l'ikan garing $(v)$ entre autre à l'épervier. Dans certains villages, on fait l'élevage des poissons rouges dans les étangs (teabat). Chacun est libre de pêcher ou chasser dans sa fédération et de cueillir les produits de la forêt, tant qu'il paie le revenu dû au radja (pantjoung alas).

Il n'y a pas de marché et fort peu de commerce. Si besoin est, on porte des poules, des œufs ou du padi aux bas-pays et fait ses emplettes avec l'argent obtenu. Avant la. guerre on vendait cela aisément aux ouvriers des plantations.

L'industrie est peu importante; on ne tisse pas mais tresse des nattes avec de beaux dessins. De temps à autre, on rencontre une forge. Il n'y a pas de mines.

Avant la guerre, la monnaie officielle était la monnaie des Indes Néerlandaises. Cependant les amendes étaient toujours infligées d'après le droit coutumier en tahils (10 tahils $=32$ dollars de Singapore). Comme mesure de longueur on connait la gantang ou depa. $( \pm 1,50 \mathrm{~m})$, un alo $(0,60 \mathrm{~m})$, un djonkal (portée du pouce au doigt moyen $\pm 0,25 \mathrm{~m}$ ). Pour le poids on emploie le datjin, et compte en pikols. La mesure de volume est le gantang (de riz) de 15 à 18 kilos, qui contient 4 soupak. Il y a peu d'uniformité, chaque radja ayant son propre gantang (v).

19. Impôts. Selon l'ancien usage, les Radjas et les chefs avaient droit à quelques journées de travail non rémunéré des indigènes; au radja revenait la défense droite d'un éléphant, la patte droite de devant d'un cerf abattu dans la chasse, chaque enfant non légitime, de même $10 \%$ du revenu de l'exploitation de la forêt, pantjoung alas, de plus l'oulou taloun $(v)$ un gantang de riz et une poule après chaque récolte du padi. En pratique ces droits n'étaient plus en usage. Quand une fille se marie, le chef a droit à l'ap:oun (v), c. à. d. 1/10 de la dot, revenu qu'il doit partager avec les datouks. Si la fille du chef se marie, ce droit revient au radja, de même dans le cas où une fille se marie dans le village où le radja réside. C'est le fiancé qui doit payer la dette. Le radja et les chefs ont droit à une partie des amendes. Si les revenus légaux sont minimes il y a par contre toute une série d'obligations vis-à-vis des chefs, surtout le devoir d'hospitalité exigé par l'adat.

20. Situation politique. Au début, le régime était l'autocratie du radja, qui avait tout pouvoir sans être responsable à personne. Il était assisté par un remplaçant, le wakil, une sorte d'ordonnance, le tongkat et le prédicateur malim, ensuite par le kerapatan et une cour de notables appelés datouks. Toutes les dignités sont héréditaires dans la lignée masculine. Dans le cas d'âge mineur, une régence est établie.

Le chef de la fédération s'appelle radjah adatouk et est élu par les radjas et les datouks. L'adat exige que chaque radja adatouk, radja, chef de village soit marié: un chef non marié ne pourrait s'acquitter de l'obligation de fournir deux repas par jour aux visiteurs étrangers (ce qui encourage le vagabondage).

Après l'élection, on arrange un repas de fête pour lequel les datouks et chefs de village apportent comme cadeaux (legit) du riz, des poules, etc. On fait part d'une nomination aux chefs des deux fédérations et ils sont invités à participer au festin. Il paraît que les chefs les plus importants étaient ceux de Napompar et de Rimbaja.

Si l'isolement de la région la soustrayait à l'influence des royaumes malais de la côte, par contre chaque radja tâchait d'agrandir son territoire. Ces disputes réitérées firent que les radjas réunis acceptèrent la souveraineté néerlandaise en 1909 à $\mathrm{Na}$ pompar: les querelles de territoire cessèrent, et en 1916, on accepta de réunir les 
deux fédérations et Bilah sous la tutelle du Toungkou Sou tan Bilah Alam Sjah, de Bilah. Les radjas n'apprécièrent pas l'intervention du Toungkou qui avait à juger de l'aptitude des candidats. En plus, du fait que les frontières n'étaient pas bien connues il résultait très souvent des disputes sur la répartition des revenues. L'établissement des plantations et des cultures occidentales, tout en augmentant les revenus, avivaient les querelles, de sorte que les relations entre ces chefs ne sont jamais devenues fort amicales.

\section{VOCABULAIRE}

Les chiffres indiquent les paragraphes du texte où figurent les expressions bataks.

Adat - droit coutumier 10; Alang alang - imperata spec. 6; Alo - mesure de longueur, environ $60 \mathrm{~cm} \mathrm{18;} \mathrm{Apioun} \mathrm{-} \mathrm{1/10} \mathrm{de} \mathrm{la} \mathrm{boli} \mathrm{ou} \mathrm{dot} \mathrm{revenant} \mathrm{au} \mathrm{chef} \mathrm{du} \mathrm{village} \mathrm{dont} \mathrm{une} \mathrm{fille} \mathrm{se} \mathrm{marie} \mathrm{19;}$ Bebei - voir malim, prédicateur musulman 12; Bétel - piper betle L. 10; Boli - dot 12; Bounga balei - arbre de vie 12; Bouroung tjindarwasi - oiseau du paradis 12; Datouk - chef de village 10; Depa - mesure de longueur (=gantang) $=11 / 2$ mètre $18 ;$ Djonkal - mesure de longueur : du pouce au majeur $\pm 25 \mathrm{~cm} \mathrm{18;} \mathrm{Djaoung,} \mathrm{djagoung} \mathrm{-} \mathrm{riz,} \mathrm{zea} \mathrm{maïs} \mathrm{L.} \mathrm{8;} \mathrm{Djaras} \mathrm{-} \mathrm{panier} \mathrm{5;} \mathrm{Gan-}$ tang - mesure de longueur $=\operatorname{depa}=1^{1} / 2$ mètre $18 ;$ Gantang - mesure de volume (riz) contenant 15 ou 18 kilos ou 4 soupak 18; Gardian - cassave, Manihot utilissima Pohl 8; Gelang (Golang) bracelets en argent ou cuivre 7; Gordang - tambour en cône 9; Hadji - musulman qui a fait le pélérinage à la Mecque 11; Hapatangan - défense de mariage 12; Horas horas - salut! 9; Houroung korong - colliers de perles 7 ; Hourabou - petites pendeloques aux oreilles 7; Idjouk, indjouk, douk - fibre du palmier, Arenga pinnata Merr. 6; Ikaiou - soupe aux légumes ou feuilles 8; Ikan garing - poisson séché 18 ; Ikan merah - poisson rouge 18 ; Indahan - riz cuit 8 ; Kati poids de 600 grammes environ 18 ; Kepala kampong - chef de village $=$ radja 20 ; Kerapatan besar - cour supérieure 13; Ketoua - le (plus) âgé 6; Koulim - bois pour la construction des bateaux, Kulim = scorodocarpus borneënsis Becc. 18; Kris - poignard 15: Ladang - terrain déblayé pour la culture 6; Lapah djagoung - bractées de maïs séchées pour y rouler du tabac 8; Lasiak - piment 8; Legit - cadeau à l'élection des chefs 20; Lombok - piment, Capsicum Annuum L. 8; Lompat pagar - mariage par enlèvement. Pagar = haie, clôture 12; Malim - voir bebei, prédicateur et instructeur mahométan 12; Malim daborou - femme qui lave les défuntes 12; Mamouro - chasser les oiseaux et bêtes sauvages 18; Mandouri - sarcler 18; Manisan bagod - suc du palmier arèn, arenga pinnata Merr. 8; Manisan tobou - Saccharum officinarum L. 8; Manoutoung - brûler les bois sec lors du déblaiement du terrain 18; Marbabo - arracher les mauvaises herbes 18; Mardega - battage du riz 18; Marga - territoire sur lequel on a un droit de disposition 11; Martaba - couper les arbres pour défricher le terrain 18; Medang, modang - bois pour montants et poteaux 18; Mendersah - maison de prière 6; Mengarabi - couper les arbustes pour déblayer le terrain 18; Meniamè - semage du riz sur le ladang 18; Meranti - bois employé pour les maisons, shorea spec. div. 18; Mertjon - feu d'artifice 15; Missigit - église musulmane 12; Momongan - le plus petit tambour 9; Montia - danse des sabres 9; Mordang - planter le riz sur le ladang 18; Niboung - oncosperma filamentosa Bl. 6; Obi kaiou - cassave, Manihot utilissima Pohl. 8; Odap - tambour 9; Ogoung - moyen tambour 9; Orang - homme 12; Ordang - plantoir 18; Oulou taloun - revenu des chefs à chaque récolte ; Oupa oupa - cérémonie païenne de bénédiction 12; Pantjoung alas - revenu de chasse, pêche et récolte forestière, dû au radja 18 ; Pantoun chanson populaire 12; Parang - couperet 15; Pedoung - sabre 15; Penghoulou - grand-prêtre musulman 6; Pinang - le sirih (piperacées à mâcher) s'offre à chaque occasion cérémonielle, Areca catechu L.10; Radja - Kepala Kampong 20; Sarong - jupe indigène 7; Sarounè - flûte 9; Sikap - planchettes pour couvrir les toits 6; Slendang - morceau de tissu long et étroit 9; Sopo - maison de la communauté 12; Soubang - grandes pendeloques aux oreilles 7; Soundout - génération 11; Soumbang inceste par mariage dans la marga 12 ; Soupak - mesure de volume : ${ }^{1 / 4}$ de gantang, 3,5 à 4,5 kilos 18 ; Sourau - maison de prière 6; Tahil - prix des amendes: 1 tahil $=32$ dollars de Singapore d'avant guerre 18; Tali sajak - cymbales 9; Tandia - lance 15; Tawah tawah - grand tambour 9; Teabat - poissons rouges 16; Tjongka - calotte couvre chef 7 ; Tongkat - courrier du chef 12 ; Toungkou prince 20; Toulak sendjata - lit: détourner l'arme. Amende payée en surplus de la dot dans un cas de mariage par enlèvement 12; Wakil - représentant du chef 20.

\section{THE FEDERATION OF RADJA NA IX AND RADJA NA X ON THE EAST-COAST OF SUMATRA}

A description is given of the territory called Radja na Sembilan and Sepuluh, a federation of 9 or 10 previously more or less autonomous areas in Sumatra. The whole region comprises about $385 \mathrm{~km}^{2}$. The terrain is mountainous, with numerous rivers and rivulets, and verv few roads; the villages are situated along the rivers; the houses are partly malayan partly Toba Batak in style. 
The people consider themselves immigrants, who came from Toba Batak country two centuries ago. Most are converted to Islamism, some to Christianism, a number of pagan rites still persist. Marriage is an important economic affair, cross cousin marriage is allowed. The dialect is connected with Angola Batak and Kualuh, for relations with foreigners Toba Batak is used. The economy is mainly agricultural, rice, maize, coffee, robber, some rotan and lacquer. A description is given of the jurisdiction and political situation up to the world war II as well as of some tribal customs.

L'auteur remercie vivement Monsieur le professeur R. A. M. BERGMAN, directeur de la section d'anthropologie physique et culturelle de l'Institut Roval des Tropiques à Amsterdam, pour l'amabilité d'avoir rédigé le texte français de cet article.

\section{NEUE GRUN DWERKE DER LANDESKUNDE ÖSTERREICHS}

In den letzten Jahren mehren sich in unserem Nachbarlande Österreich die wissenschaftlichen landeskundlichen Publikationen, die deutlich das Bestreben erkennen lassen, ein geschlossenes Bild der Heimat zu entwerfen, um dem Forscher wie dem Lehrer und Praktiker eine Grundlage für seine Arbeit, dem Bürger einen Einblick in seine Umwelt zu geben.

In den Jahren 1952-53 erschienen, herausgegeben vom Österreichischen Statistischen Zentralamt, drei Publikationen, in denen das reichhaltige statistische Material, das sich insbesondere aus der Volkszählung 1951 ergeben hatte, verarbeitet wurde, um in großen Zügen ein Bild der Struktur Österreichs zu geben. Als erste Publikation wurde 1952 ein „Verwaltungsatlas auf statistischer Grundlage "1 herausgegeben, der in erster Linie ein Hilfsbuch für Österreichs Verwaltung darstellt, nicht minder aber auch für den Geographen interessant ist. Zur Veranschaulichung der zahlenmäßigen Darstellungen dienen hauptsïchlich Karten, denen die Veröffentlichung ihre Bezeichnung als Atlas verdankt. Unter den zahlreichen Kapiteln seien nur die folgenden erwähnt, die insbesondere für den Geographen Interesse besitzen: Verfassung, geographische Angaben, Bevölkerungsentwicklung 1901-1951, politische Einteilung des Landes, Straßennetz, Bahnnetz, Waldverbreitung, Bodenschätze und zahlreiche andere mehr. Ebenfalls äußerst reichhaltiges Material enthält die 1953 erschienene Publikation „Österreichs Berölkerung in Bild und Zahl"2. Um die Hauptergebnisse der Volkszählung 1951 für ganz Österreich möglichst ohne Zeitverlust der Öffentlichkeit zugänglich zu machen, wurden sie in gedrängter Form tabellarisch und graphisch dargestellt, sodaß sich die vorliegende Veröffentlichung besonders auch für den Ausländer sehr gut zur Orientierung eignet. Aus den Tabellen kann entnommen werden, daß Österreich 1951 eine Bevölkerung von 6933905 Seelen aufwies gegenüber 6760233 im Jahre 1934, woraus für 1951 eine Bevölkerungsdichte von 83 resultiert. Die Hauptanteile entfallen auf die vier Bundesländer Wien, Nieder-, Oberösterreich und Steiermark, die alle über eine Million Einwohner besitzen, während die restlichen, auch flächenmäßig kleineren Bundesländer alle weniger als eine halbe Million ausweisen. Weitere Tabellen gliedern die Einwohnerschaft nach Meereshöhe, Alter und Familienstand, Religion, Staatsangehörigkeit, Bildung und Erwerbstätigkeit. Den $38 \%$ in Industrie und Gewerbe Beschäftigten stehen $22 \%$ der Land- und Forstwirtschaft gegenüber, während sich der Rest auf Handel und Verkehr, freie Berufe, öffentlichen Dienst und häusliche Dienste in dieser Reihenfolge verteilt. Als dritte Publikation dieser Reihe erschien ebenfalls 1953 der Band „Österreichs Landwirtschaft in Bild und $Z a h l^{\star 3}$, wobei in analoger Weise Stand und Entwicklung der österreichischen Landwirtschaft auf Grund der neuesten statistischen Ergebnisse aufgezeigt werden. Im ersten Teil werden Witterungsablauf und Bodentypen untersucht, während der zweite, überwiegende Teil der landwirtschaftlichen Produktion gewidmet ist. Wir erfahren dabei unter anderem, daß von den 8201463 ha Wirtschaftsfläche Österreichs $195249,8 \%$ landwirtschaftlich und $36,9 \%$ forstwirtschaftlich genutzt wurden und die restlichen 13,3\% unproduktive Flächen darstellen. 40,5\% der landwirtschaftlichen Fläche werden als Acker, 34,7\% als Wiese und Weide und 22,5 $\%$ als "Alpines Grünland" genutzt; der Rest entfällt auf Wein- und Obstgärten.

Diese recht summarischen Angaben aus dem Inhalt der drei stattlichen Bände können naturgemäß nur einen kleinen Ausschnitt aus dem reichhaltigen Material bilden, dessen Wert noch dadurch verstärkt wird, daß die Zahlen übersichtlich zusammengefaßt sind und dadurch rasch miteinander verglichen werden können. Die zahlreichen Illustrationen, vorwiegend instruktive graphische Darstellungen, helfen mit, den Publikationen einen großen informatorischen Wert, insbesondere auch für den Ausländer zu geben.

1 Wien 1952, 51 Seiten, 30 Tafeln.

2 Wien 1953, 51 Seiten, 39 Tafeln.

3 Wien 1953, 118 Seiten, 60 Tafeln, 13 Abbildungen. 